\title{
One-sided discrete square function
}

by

\author{
A. DE LA Torre (Málaga) and J. L. Torrea (Madrid) \\ Dedicated to Professor Carlos Segovia on his 65th birthday
}

\begin{abstract}
Let $f$ be a measurable function defined on $\mathbb{R}$. For each $n \in \mathbb{Z}$ we consider the average $A_{n} f(x)=2^{-n} \int_{x}^{x+2^{n}} f$. The square function is defined as$$
S f(x)=\left(\sum_{n=-\infty}^{\infty}\left|A_{n} f(x)-A_{n-1} f(x)\right|^{2}\right)^{1 / 2} .
$$

The local version of this operator, namely the operator

$$
S_{1} f(x)=\left(\sum_{n=-\infty}^{0}\left|A_{n} f(x)-A_{n-1} f(x)\right|^{2}\right)^{1 / 2},
$$

is of interest in ergodic theory and it has been extensively studied. In particular it has been proved [3] that it is of weak type $(1,1)$, maps $L^{p}$ into itself $(p>1)$ and $L^{\infty}$ into BMO. We prove that the operator $S$ not only maps $L^{\infty}$ into BMO but it also maps BMO into BMO. We also prove that the $L^{p}$ boundedness still holds if one replaces Lebesgue measure by a measure of the form $w(x) d x$ if, and only if, the weight $w$ belongs to the $A_{p}^{+}$ class introduced by E. Sawyer [8]. Finally we prove that the one-sided Hardy-Littlewood maximal function maps BMO into itself.
\end{abstract}

Introduction. Let $f$ be a measurable function defined on $\mathbb{R}$. For each $n \in \mathbb{Z}$ define the operator $A_{n}$ by

$$
A_{n} f(x)=\frac{1}{2^{n}} \int_{x}^{x+2^{n}} f(y) d y
$$

It is a classical problem to study the different kinds of convergence of the sequence $\left\{A_{n} f\right\}_{n}$ when the function $f$ belongs to $L^{p}(\mathbb{R}, d x), p$ being in the

2000 Mathematics Subject Classification: Primary 42B20; Secondary 42B25.

Key words and phrases: square function, weights, BMO.

The first author was supported by Ministerio de Ciencia y Tecnología BFM 2001-1638 and Junta de Andalucia. The second author was supported by European Comission via the TMR network "Harmonic Analysis". 
range $1 \leq p<\infty$. A method of measuring the speed of convergence of this sequence is to analyze the boundedness of the square function

$$
S f(x)=\left(\sum_{n=-\infty}^{\infty}\left|A_{n} f(x)-A_{n-1} f(x)\right|^{2}\right)^{1 / 2} .
$$

Recently, among other operators, the local version of this operator, namely the operator

$$
S_{1} f(x)=\left(\sum_{n=-\infty}^{0}\left|A_{n} f(x)-A_{n-1} f(x)\right|^{2}\right)^{1 / 2}
$$

has been studied in [3] and [4]. It has been proved that $S_{1}$ maps $L^{p}(\mathbb{R}, d x)$ into itself for each $p$ in the range $1<p<\infty$ and that $S_{1}$ is of weak type $(1,1)$, that is,

$$
|\{x: S f(x)>\lambda\}| \leq \frac{C}{\lambda} \int_{\mathbb{R}}|f(y)| d y,
$$

where as usual we denote by $|E|$ the Lebesgue measure of a set $E \subset \mathbb{R}$.

The aim of this note is to characterize the weights $\omega$ (almost everywhere positive measurable functions) such that either, for each $p$ in the range $1<p<\infty$, the operator $S$ maps $L^{p}(\mathbb{R}, \omega(x) d x)$ into itself, or the following weak type $(1,1)$ inequality is satisfied:

$$
\omega(\{x: S f(x)>\lambda\}) \leq \frac{C}{\lambda} \int_{\mathbb{R}}|f(y)| \omega(y) d y,
$$

where $C$ is a positive constant.

In our opinion, the natural way of proving weighted results for this operator is to use the theory of vector-valued singular integrals. Therefore it would seem that the right class of weights were the $A_{p}$ classes of Muckenhoupt, but this would overlook the fact that the operator $S$ is one-sided, i.e. $S f(x)=S\left(f(\cdot) \chi_{(x, \infty)}(\cdot)\right)(x)$; clearly $A_{p}$ is not a necessary condition. For one-sided operators the natural classes are the $A_{p}^{+}$classes introduced by E. Sawyer [8] (see (2.1) and (2.2) in Section 2 for the corresponding definitions). In fact we shall prove the following result.

TheOREM A. Given $p$ in the range $1 \leq p<\infty$, and a weight $\omega$, the following are equivalent:

(i) There exists a constant $C_{p}$ such that

$$
(\omega(\{x: S f(x)>\lambda\}))^{1 / p} \leq \frac{C_{p}}{\lambda}\|f\|_{L^{p}(\mathbb{R}, \omega(x) d x)},
$$

(ii) $\omega \in A_{p}^{+}$.

Moreover, in the case $1<p<\infty$ they are also equivalent to the following statement: 
(iii) There exists a constant $C_{p}$ such that

$$
\|S f\|_{L^{p}(\mathbb{R}, \omega(x) d x)} \leq C_{p}\|f\|_{L^{p}(\mathbb{R}, \omega(x) d x)} .
$$

In order to prove this theorem we shall introduce a one-sided vectorvalued Calderón-Zygmund operator $U$ (see Definition 1.1). We believe that our main contribution is a careful geometric analysis of the kernel of the operator $U$ (see Lemma 1.2 and also (1.4)) that allows us to show that the kernel satisfies some one-sided Hörmander type conditions (see Definition 1.5). Conditions of this type suggest in general boundedness from $L^{\infty}$ into BMO; however, the following example seems to forbid such a result.

EXAMPLE. If $f=\sum_{n=0}^{\infty} \chi_{\left[4^{n}, 2 \cdot 4^{n}\right]}=\sum_{i=0}^{\infty} \chi_{\left[2^{2 i}, 2^{2 i+1}\right]}$, then $S f(x)=\infty$ for every $x$.

Proof. We shall see that $S f(0)=\infty$; if $x \neq 0$ one can prove $S f(x)=$ $\infty$ in the same way. If $j=2 k+1$ then $A_{j} f(0)=\left(1 / 2^{j}\right) \sum_{i=0}^{k} 4^{i}$, while $A_{j+1} f(0)=\frac{1}{2} A_{j} f(0)$. It follows that $\left|A_{2 k+2} f(0)-A_{2 k+1} f(0)\right|=\frac{1}{2} A_{2 k+1} f(0)$ $>\frac{1}{4}$ and so $\stackrel{S}{S} f(0)=\infty$.

In fact as a byproduct of our study we shall obtain the following dichotomy results that we believe are of independent interest.

Theorem B. (a) Given a function $f$ in $L^{\infty}(\mathbb{R})$, either $S f(x)=\infty$ for a.e. $x$ or $S f(x)<\infty$ for a.e. $x$. Moreover in the second case $S f \in \mathrm{BMO}$ and there exists a constant $C$ such that $\|S f\|_{\mathrm{BMO}(\mathbb{R})} \leq C\|f\|_{L^{\infty}(\mathbb{R})}$.

(b) Given a function $f$ in $\mathrm{BMO}(\mathbb{R})$, either $S f(x)=\infty$ for a.e. $x$ or $S f(x)<\infty$ for a.e. $x$. Moreover in the second case $S f \in \mathrm{BMO}$ and there exists a constant $C$ such that $\|S f\|_{\mathrm{BMO}(\mathbb{R})} \leq C\|f\|_{\mathrm{BMO}(\mathbb{R})}$.

We believe that the geometric analysis developed for the study of the square function can be of interest for other one-sided operators. In particular we apply these ideas to study the behaviour of the one-sided HardyLittlewood maximal operator acting on functions that belong to the BMO class, and again we get a dichotomy result of the type of Theorem B (see Theorem 3.10).

The organization of the paper is as follows. In Section 1 we develop the adapted one-sided Calderón-Zygmund theory that we need and as a quick consequence we prove Theorem B. Section 2 is devoted to the study of weighted inequalities, and in particular to the proof of Theorem A. Finally in Section 3 we analyze the one-sided Hardy-Littlewood maximal operator. We end this introduction with some notation. Given a measurable set $E$ and a weight $w, w(E)$ will represent the integral of $w$ on $E$. If $I$ is an interval and $f$ a locally integrable function, we will denote by $f_{I}$ the average of $f$ on $I$, i.e. $f_{I}=(1 /|I|) \int_{I} f$. 


\section{Vector-valued analysis. Boundedness on BMO and $L_{\infty}$}

Definition 1.1. Given a locally integrable function $f$ we define the sequence-valued operator $U$ as follows:

$$
\begin{aligned}
U f(x) & =\left\{A_{n} f(x)-A_{n-1} f(x)\right\}_{n} \\
& =\left\{\int_{\mathbb{R}}\left(\frac{1}{2^{n}} \chi_{\left(-2^{n}, 0\right)}(x-y)-\frac{1}{2^{n-1}} \chi_{\left(-2^{n-1}, 0\right)}(x-y)\right) f(y) d y\right\}_{n} \\
& =\int_{\mathbb{R}} K(x-y) f(y) d y,
\end{aligned}
$$

where $K$ is the sequence-valued function

$$
K(x)=\left\{\frac{1}{2^{n}} \chi_{\left(-2^{n}, 0\right)}(x)-\frac{1}{2^{n-1}} \chi_{\left(-2^{n-1}, 0\right)}(x)\right\}_{n} .
$$

Observe that $\|U f(x)\|_{\ell^{2}}=S f(x)$. Although the operator $U$ is defined in terms of averages with nonsmooth kernels it satisfies a one-sided smoothness condition, which will play the role of the Hörmander condition in the classical theory of singular integrals.

Lemma 1.2. Given $x_{0} \in \mathbb{R}$ and $i \in \mathbb{Z}$, consider $x$ and $y$ in $\mathbb{R}$ such that $x_{0}<x \leq x_{0}+2^{i}$ and $x_{0}+2^{j}<y \leq x_{0}+2^{j+1}$ with $j>i$. Let $\chi_{n}(y)=\chi_{\left(-2^{n}, 0\right)}(y)$. Then $\chi_{n}(x-y)-\chi_{n}\left(x_{0}-y\right)=0$ unless $n=j$ in which case $\chi_{j}(x-y)-\chi_{j}\left(x_{0}-y\right)=\chi_{\left(x_{0}+2^{j}, x+2^{j}\right)}(y)$.

Proof. It is clear that $\chi_{n}(x-y)=\chi_{\left(x, x+2^{n}\right)}(y)$. Now if $n<i$ then $x+2^{n}<x-x_{0}+x_{0}+2^{i} \leq x_{0}+2^{i+1} \leq x_{0}+2^{j}<y$. Therefore $\chi_{n}(x-y)=0$. Obviously the same holds for $\chi_{n}\left(x_{0}-y\right)$. If $i \leq n<j$ then $x+2^{n} \leq$ $x_{0}+2^{i}+2^{n} \leq x_{0}+2 \cdot 2^{n} \leq x_{0}+2^{j}$, and $\chi_{n}(x-y)=\chi_{n}\left(x_{0}-y\right)=0$. If $n>j$ then $x+2^{n}>x_{0}+2^{n} \geq x_{0}+2^{j+1} \geq y$, and since $y>x>$ $x_{0}$ we have $\chi_{n}(x-y)-\chi_{n}\left(x_{0}-y\right)=1-1=0$. Finally if $n=j$ then $\chi_{j}\left(x_{0}-y\right)=\chi_{\left(x_{0}, x_{0}+2^{j}\right)}(y)=0$, while $\chi_{j}(x-y)=\chi_{\left(x, x+2^{j}\right)}(y)=1$ whenever $x_{0}+2^{j} \leq y \leq x+2^{j}$.

Lemma 1.3 (Smoothness condition). Assume $x_{0}, x, y$ are as in the preceding lemma. Let $K$ be the vector-valued kernel that appears in Definition 1.1. Then

$$
\left\|K(x-y)-K\left(x_{0}-y\right)\right\|_{\ell^{2}}=\frac{\sqrt{2}}{2^{j}} \chi_{\left(x_{0}+2^{j}, x+2^{j}\right.}(y) .
$$

Proof. We have

$$
\begin{aligned}
\left\|K(x-y)-K\left(x_{0}-y\right)\right\|_{\ell^{2}}^{2}= & \sum_{n} \mid \frac{1}{2^{n}} \chi_{n}(x-y)-\frac{1}{2^{n-1}} \chi_{n-1}(x-y) \\
& -\left.\left(\frac{1}{2^{n}} \chi_{n}\left(x_{0}-y\right)-\frac{1}{2^{n-1}} \chi_{n-1}\left(x_{0}-y\right)\right)\right|^{2}
\end{aligned}
$$




$$
\begin{aligned}
= & \sum_{n} \mid \frac{1}{2^{n}} \chi_{n}(x-y)-\frac{1}{2^{n}} \chi_{n}\left(x_{0}-y\right) \\
& -\left.\left(\frac{1}{2^{n-1}} \chi_{n-1}(x-y)-\frac{1}{2^{n-1}} \chi_{n-1}\left(x_{0}-y\right)\right)\right|^{2} \\
= & 2\left|\frac{1}{2^{j}} \chi_{j}(x-y)-\frac{1}{2^{j}} \chi_{j}\left(x_{0}-y\right)\right|^{2}=2\left|\frac{1}{2^{j}} \chi_{\left(x_{0}+2^{j}, x+2^{j}\right)}(y)\right|^{2}
\end{aligned}
$$

It follows from (1.4) that the kernel $K$ does not satisfy the "gradient" condition

$$
\left\|K(x-y)-K\left(x_{0}-y\right)\right\|_{\ell^{2}} \leq C\left(x-x_{0}\right)\left(y-x_{0}\right)^{-2}
$$

whenever $y-x_{0}>2\left(x-x_{0}\right)$. Nevertheless (1.4) will allow us to prove some kind of condition that implies Hörmander's.

Parallel to [7] we give the following

Definition 1.5. We say that the kernel $K$ satisfies one-sided condition $D_{r}$, for $1 \leq r<\infty$, and write $K \in D_{r}$, if there exists a sequence $\left\{c_{l}\right\}_{l=1}^{\infty}$ of positive numbers such that $\sum_{l} c_{l}<\infty$ and for any $l \geq 2$ and $x>0$,

$$
\left(\int_{S_{l}(x)}\|K(x-y)-K(-y)\|_{\ell^{2}}^{r} d y\right)^{1 / r} \leq c_{l}\left|S_{l}(x)\right|^{-1 / r^{\prime}},
$$

where $S_{l}(x)=\left(2^{l} x, 2^{l+1} x\right)$.

It is easy to see that $D_{s} \subset D_{r} \subset D_{1}$ for $1 \leq r<s$, where $K \in D_{1}$ means the following Hörmander's type condition:

$$
\int_{\{y>4 x\}}\|K(x-y)-K(-y)\|_{\ell^{2}} d y \leq C
$$

where $C$ is a positive constant.

Theorem 1.6. The kernel $K$ introduced in Definition 1.1 satisfies $D_{r}$ for any $r \geq 1$ with $c_{l}=C 2^{-l / r}$.

Proof. Given $x$, choose an integer $i$ such that $2^{i-1} \leq x<2^{i}$. Lemma 1.3 and Hölder's inequality give us

$$
\begin{aligned}
\left(\int_{2^{l} x}^{2^{l+1} x}\|K(x-y)-K(-y)\|_{\ell^{2}}^{r} d y\right)^{1 / r} & \\
& \leq\left(\int_{2^{l+i-1}}^{2^{l+i}}\|K(x-y)-K(-y)\|_{\ell^{2}}^{r} d y\right)^{1 / r}
\end{aligned}
$$




$$
\begin{gathered}
+\left(\int_{2^{l+i}}^{2^{l+i+1}}\|K(x-y)-K(-y)\|_{\ell^{2}}^{r} d y\right)^{1 / r} \\
\leq 2 \frac{2^{i / r}}{2^{l+i}}=C 2^{-l / r}\left|S_{l}(x)\right|^{-1 / r^{\prime}} .
\end{gathered}
$$

Since we have Hörmander's condition and it is easy to check that the Fourier transform of the kernel of our vector-valued operator $U$ is bounded we deduce that the operator $S$ is bounded on $L^{p}, p>1$, and satisfies a weak type $(1,1)$ inequality. Now we shall use our smoothness condition in order to study the pointwise size of the operator. We start with the following technical lemma.

LEMMA 1.7. Let $f$ be a locally integrable function such that $S f\left(x_{0}\right)<\infty$ for some $x_{0} \in \mathbb{R}$. Then $S\left(f(\cdot) \chi_{[x, \infty)}(\cdot)\right)\left(x_{0}\right)<\infty$ for any $x>x_{0}$.

Proof. Let $i$ be an integer such that $2^{i}<x-x_{0} \leq 2^{i+1}$, consider $g=$ $f \chi_{(x, \infty)}$ and $\mathcal{I}=\int_{x_{0}}^{x} f$. If $j \leq i$, then $A_{j} g\left(x_{0}\right)=0$. If $j>i$ then

$$
A_{j} g\left(x_{0}\right)=\frac{1}{2^{j}} \int_{x_{0}}^{x_{0}+2^{j}} g=\frac{1}{2^{j}} \int_{x_{0}}^{x_{0}+2^{j}} f-\frac{1}{2^{j}} \int_{x_{0}}^{x} f=A_{j} f\left(x_{0}\right)-\frac{1}{2^{j}} \mathcal{I} .
$$

Therefore

$$
\begin{aligned}
S g\left(x_{0}\right)= & \left(\sum_{j}\left|A_{j} g\left(x_{0}\right)-A_{j-1} g\left(x_{0}\right)\right|^{2}\right)^{1 / 2} \\
= & \left|A_{i+1} f\left(x_{0}\right)-\frac{1}{2^{i+1}} \mathcal{I}\right|+\left(\sum_{j>i+2}\left|A_{j} f\left(x_{0}\right)-A_{j-1} f\left(x_{0}\right)\right|^{2}\right)^{1 / 2} \\
& +\left(\sum_{j>i+2}\left|\frac{1}{2^{j-1}}-\frac{1}{2^{j}}\right|^{2}\right)^{1 / 2} \mathcal{I} \\
\leq & \left|A_{i+1} f\left(x_{0}\right)-\frac{1}{2^{i+1}} \mathcal{I}\right|+S f\left(x_{0}\right)+\left(\sum_{j>i+2}\left|\frac{1}{2^{j-1}}-\frac{1}{2^{j}}\right|^{2}\right)^{1 / 2} \mathcal{I} \\
< & \infty .
\end{aligned}
$$

Proposition 1.8. Let $f \in L^{\infty}(\mathbb{R})$ and let $x_{0}$ be such that $S f\left(x_{0}\right)<\infty$. Then $S f(x)<\infty$ for almost all $x>x_{0}$.

Proof. We shall prove that the $\ell^{2}$-valued operator $U$ defined in 1.1 satisfies $\|U f(x)\|_{\ell^{2}}<\infty$ for almost every $x>x_{0}$. Consider the interval $I_{0}=$ $\left(x_{0}, x_{0}+4\left(x-x_{0}\right)\right)$. Let $f_{1}=f \chi_{I_{0}}$ and $f_{2}=f-f_{1}$. Since the operator $S$ is bounded on $L^{2}$, we have $\left\|U f_{1}(y)\right\|_{\ell^{2}}=S f_{1}(y)<\infty$. On the other hand, by using the one-sided nature of $S$ and the last Lemma 1.7, we deduce that 
for $x>x_{0}$,

$$
\left\|U f_{2}\left(x_{0}\right)\right\|_{\ell^{2}}=S f_{2}\left(x_{0}\right)=S\left(f(\cdot) \chi_{\left(x_{0}+4\left(x-x_{0}\right), \infty\right)}(\cdot)\right)\left(x_{0}\right)<\infty .
$$

Therefore it is enough to prove that $\left\|U f_{2}(x)-U f_{2}\left(x_{0}\right)\right\|_{\ell^{2}}<\infty$. By using again the one-sided nature and condition $D_{r}$ with $r=1$, we obtain

$$
\begin{array}{r}
\left\|U f_{2}(x)-U f_{2}\left(x_{0}\right)\right\|_{\ell^{2}}=\left\|\int_{x_{0}+4\left(x-x_{0}\right)}^{\infty}\left(K(x-y)-K\left(x_{0}-y\right)\right) f(y) d y\right\|_{\ell^{2}} \\
\leq \int_{x_{0}+4\left(x-x_{0}\right)}^{\infty}\left\|K(x-y)-K\left(x_{0}-y\right)\right\| \ell_{\ell^{2}}|f(y)| d y \leq C\left\|_{f}\right\|_{\infty} .
\end{array}
$$

Proposition 1.9. Let $f \in L^{\infty}(\mathbb{R})$ and let $x_{0}$ be such that $S f\left(x_{0}\right)<\infty$. Then $S f(y)<\infty$ for almost all $y<x_{0}$.

Proof. Following the proof of the last proposition, we shall see that $\|U f(y)\|_{\ell^{2}}<\infty$ for almost every $y<x_{0}$. Given $y<x_{0}$. Set $I_{0}=(y, y+$ $\left.4\left(x_{0}-y\right)\right), f_{1}=f \chi_{I_{0}}$ and $f_{2}=f-f_{1}$. Again $\left\|U f_{1}(y)\right\|_{\ell^{2}}=S f_{1}(y)<\infty$ for almost every $y$, because $S$ is bounded on $L^{2}$. On the other hand, by using the one-sided nature of $S$ and Lemma 1.7 (observe that $y+4\left(x_{0}-y\right)>x_{0}$ ), we see that for $y<x_{0}$,

$$
\left\|U f_{2}\left(x_{0}\right)\right\|_{\ell^{2}}=S f_{2}\left(x_{0}\right)=S\left(f(\cdot) \chi\left(y+4\left(x_{0}-y\right), \infty\right)(\cdot)\right)\left(x_{0}\right)<\infty .
$$

Therefore it is enough to prove that $\left\|U f_{2}(y)-U f_{2}\left(x_{0}\right)\right\|_{\ell^{2}}<\infty$. Now the proof ends as in the last proposition.

We have proved that for an $L^{\infty}$ function $f, S f$ is either infinite a.e. or finite a.e. The same result can be proved, with minor modifications, for BMO functions. Therefore in order to prove Theorem B we need to prove that for functions $f$ in $L^{\infty}$ (respectively in BMO) with $S f$ finite almost everywhere, the function $S f$ is in BMO, and the BMO norm of $S f$ is controlled by the $L^{\infty}$ norm (respectively the BMO norm) of $f$. We shall give only the proof in the case $f \in$ BMO. The case $f \in L^{\infty}$ is easier and we leave the details to the reader. We start with a technical lemma.

LEMMA 1.10. Let $C$ be a positive constant and let $I_{1}$ and $I_{2}$ be two intervals such that if $J$ is the smallest interval that contains both then $|J| \leq$ $C\left|I_{i}\right|, i=1,2$. Then given a function $f \in \mathrm{BMO}$ we have

$$
\left|f_{I_{1}}-f_{I_{2}}\right| \leq 2 C\|f\|_{\mathrm{BMO}} \text {. }
$$

Proof. It is clear that $\left|f_{I_{1}}-f_{I_{2}}\right| \leq\left|f_{I_{1}}-f_{J}\right|+\left|f_{J}-f_{I_{2}}\right|$. Now

$$
\left|f_{I_{1}}-f_{J}\right| \leq \frac{C}{|J|} \int_{J}\left|f-f_{J}\right| \leq C\|f\|_{\mathrm{BMO}}
$$

The other term is handled in the same way. 
Corollary 1.11. Let $f$ be $a$ BMO function, $x_{0} \in \mathbb{R}, h>0, i$ an integer such that $2^{i} \leq h<2^{i+1}, j$ any integer greater than $i$, and $I=\left(x_{0}, x_{0}+h\right)$. Then

$$
\left(\frac{1}{2^{j}} \int_{x_{0}+2^{j}}^{x_{0}+2^{j+1}}\left|f(y)-f_{I}\right|^{2} d y\right)^{1 / 2} \leq C(j-i+1)\|f\|_{\mathrm{BMO}}
$$

Proof. For any integer $l$ between $i$ and $j$ we denote by $I_{l}$ the interval $\left(x_{0}+2^{l}, x_{0}+2^{l+1}\right)$. Then

$$
\begin{aligned}
\left(\frac{1}{2^{j}} \int_{x_{0}+2^{j}}^{x_{0}+2^{j+1}}\left|f(y)-f_{I}\right|^{2} d y\right)^{1 / 2} \leq & \left(\frac{1}{2^{j}} \int_{x_{0}+2^{j}}^{x_{0}+2^{j+1}}\left|f(y)-f_{I_{j}}\right|^{2} d y\right)^{1 / 2} \\
& +\sum_{l=i+1}^{j}\left(\left|f_{I_{l}}-f_{I_{l-1}}\right|+\left|f_{I_{i+1}}-f_{I}\right|\right) .
\end{aligned}
$$

By John-Nirenberg,

$$
\left(\frac{1}{2^{j}} \int_{x_{0}+2^{j}}^{x_{0}+2^{j+1}}\left|f(y)-f_{I_{j}}\right|^{2} d y\right)^{1 / 2} \leq C\|f\|_{\mathrm{BMO}}
$$

and by the preceding lemma, each of the other terms is dominated by $4\|f\|_{\text {BMO }}$.

THEOREM 1.12. Let $f$ be a BMO function such that $S f(x)<\infty$ a.e. Then $S f \in \mathrm{BMO}$ and there exists $C$ so that

$$
\|S f\|_{\mathrm{BMO}} \leq C\|f\|_{\mathrm{BMO}}
$$

Proof. Fix $x_{0}$ and $h>0$. Consider the interval $I=\left(x_{0}, x_{0}+h\right)$ and the average $f_{I}=(1 / h) \int_{I} f$. Since $S f(x)$ is finite a.e., it is enough to prove that there exists a positive constant $C$ so that

$$
\frac{1}{h} \int_{x_{0}}^{x_{0}+h}\left|S f(x)-S\left(\left(f(\cdot)-f_{I}\right) \chi_{\left(x_{0}+8 h, \infty\right)}(\cdot)\right)\left(x_{0}\right)\right| d x \leq C\|f\|_{\mathrm{BMO}} .
$$

We define $f_{1}=\left(f-f_{I}\right) \chi_{\left(x_{0}, x_{0}+8 h\right)}$ and $f_{2}=\left(f-f_{I}\right) \chi_{\left(x_{0}+8 h, \infty\right)}$; then $f=f_{1}+f_{2}+f_{I}$. By using the linear operator $U$ defined in 1.1, we have

$$
\begin{aligned}
\frac{1}{h} \int_{x_{0}}^{x_{0}+h}\left|S f(x)-S f_{2}\left(x_{0}\right)\right| d x & =\frac{1}{h} \int_{x_{0}}^{x_{0}+h}\left|\|U f(x)\|_{\ell^{2}}-\left\|U f_{2}\left(x_{0}\right)\right\|_{\ell^{2}}\right| d x \\
& \leq \frac{1}{h} \int_{x_{0}}^{x_{0}+h}\left\|U f(x)-U f_{2}\left(x_{0}\right)\right\|_{\ell^{2}} d x \\
& =\frac{1}{h} \int_{x_{0}}^{x_{0}+h}\left\|U f_{1}(x)+U f_{2}(x)-U f_{2}\left(x_{0}\right)\right\|_{\ell^{2}} d x
\end{aligned}
$$




$$
\begin{aligned}
\leq & \frac{1}{h} \int_{x_{0}}^{x_{0}+h}\left\|U f_{1}(x)\right\|_{\ell^{2}} d x \\
& +\frac{1}{h} \int_{x_{0}}^{x_{0}+h}\left\|U f_{2}(x)-U f_{2}\left(x_{0}\right)\right\|_{\ell^{2}} d x \\
= & B_{1}+B_{2} .
\end{aligned}
$$

The boundedness of $S$ in $L^{2}$ and the John-Nirenberg inequality imply that

$$
\begin{aligned}
B_{1} & \leq\left(\frac{1}{h} \int_{x_{0}}^{x_{0}+h}\left|S f_{1}\right|^{2}\right)^{1 / 2} \leq C\left(\frac{1}{h} \int_{\mathbb{R}}\left|f_{1}\right|^{2}\right)^{1 / 2} \\
& =C\left(\frac{1}{h} \int_{x_{0}}^{x_{0}+8 h}\left|f-f_{I}\right|^{2}\right)^{1 / 2} \leq C\|f\|_{\text {BMO }} .
\end{aligned}
$$

For $B_{2}$ we just observe that if $i$ is an integer such that $2^{i} \leq h<2^{i+1}$, then using our smoothness condition and Corollary 1.11 we have

$$
\begin{aligned}
B_{2} \leq & \int_{x_{0}+8 h}^{\infty}\left\|\left(K(x-y)-K\left(x_{0}-y\right)\right)\left(f(y)-f_{I}\right)\right\|_{\ell^{2}} d y \\
\leq & \sum_{j=i+3}^{\infty}\left(\int_{x_{0}+2^{j}}^{x_{0}+2^{j+1}}\left\|K(x-y)-K\left(x_{0}-y\right)\right\|_{\ell^{2}}^{2} d y\right)^{1 / 2} \\
& \times\left(\frac{1}{2^{j}} \int_{x_{0}+2^{j}}^{x_{0}+2^{j+1}}\left|f(y)-f_{I}\right|^{2} d y\right)^{1 / 2} 2^{j / 2} \\
\leq & C \sum_{j=i+3}^{\infty} 2^{i / 2}(j-i) \frac{1}{2^{j}} 2^{j / 2}\|f\|_{\mathrm{BMO}} \leq C\|f\|_{\mathrm{BMO}} .
\end{aligned}
$$

2. Weights for the operator $S$. We recall that the $A_{p}^{+}$classes were introduced by E. Sawyer [8] in the study of the one-sided Hardy-Littlewood maximal operators

$$
M^{+} f(x)=\sup _{h>0} \int_{x}^{x+h}|f|, \quad M^{-} f(x)=\sup _{h>0} \int_{x-h}^{x}|f| .
$$

He proved the following.

Theorem. If $p>1$ then the inequality

$$
\int_{\mathbb{R}} M^{+} f(x)^{p} w(x) d x \leq C \int_{\mathbb{R}}|f(x)|^{p} w(x) d x
$$

holds for all $f \in L^{p}(w)$ if and only if $w$ satisfies the following condition: 
$\left(A_{p}^{+}\right) \quad$ There exists $C$ such that for any three points $a<b<c$,

$$
\left(\int_{a}^{b} w\right)^{1 / p}\left(\int_{b}^{c} w^{1-p^{\prime}}\right)^{1 / p^{\prime}} \leq C(c-a) \quad\left(p+p^{\prime}=p p^{\prime}\right) .
$$

If $p=1$ then the weak type inequality

$$
\int_{\left\{x: M^{+} f(x)>\lambda\right\}} w \leq \frac{C}{\lambda} \int|f(x)| w(x) d x
$$

holds for all $f \in L^{1}(w)$ if and only if

$\left(A_{1}^{+}\right) \quad$ There exists $C$ such that for almost every $x$,

$$
M^{-} w(x) \leq C w(x) .
$$

REMARK 2.3. It is known (see [6]) that condition $A_{p}^{+}$is equivalent to the following condition, called $\widetilde{A}_{p}^{*}$ :

$$
\int_{b-2 h}^{b-h} w\left(\int_{b}^{b+h} w^{1-p^{\prime}}\right)^{p-1} \leq C h^{p} .
$$

Analogously in the case $p=1$, condition $A_{1}^{+}$is equivalent to the following condition: there exists $C$, which depends on the constant in $(2.2)$, so that for almost every $b$, if $I=(a, b)$ then

$$
\int_{I} w \leq C|I| \inf \{w(x): x \in(b, 2 b-a)\} .
$$

Of course there are similar results for the operator $M^{-}$reversing the orientation of $\mathbb{R}$. Since it is easy to see that any increasing function satisfies $A_{1}^{+}$, it is obvious that the $A_{p}^{+}$classes are different from the Muckenhoupt $A_{p}$ classes. It also follows that the $A_{p}^{+}$weights do not satisfy the doubling condition nor the reverse Hölder inequality. Nevertheless there are nice substitutes for the doubling condition and the reverse Hölder inequality. Sawyer [8] proves that if $w \in A_{1}^{+}$, then $w^{r} \in A_{1}^{+}$for some $r>1$, and that $w \in A_{p}^{+}$ for some $p>1$ implies $w \in A_{s}^{+}$for some $1<s<p$. On the other hand it is easy to see that any $A_{p}^{+}$weight $w$ satisfies the following one-sided doubling condition:

There exists $C$ such that if $I=(a, b)$ and $I^{+}=(b, c)$ with $b-a=c-b$ then $\int_{I} w \leq C \int_{I^{+}} w$, which is clearly equivalent to $\int_{I \cup I^{+}} w \leq C \int_{I^{+}} w$ for some constant $C$.

In this section we shall prove Theorem A. The result does not follow from [1] because although our operator can be considered as a one-sided (vector-valued) singular integral, it does not satisfy the gradient condition 
nor the cancellation conditions assumed in that article. Our main tool will be the following extrapolation theorem of Macías and Riveros [5]:

THEOREM (Extrapolation). Let $T$ be a sublinear operator with the following property: For every $w$ such that $w^{-1} \in A_{1}^{-}$there exists a constant $C(w)$, which may depend on $w$, such that

$$
\left\|w \chi_{[x-h, x]}\right\|_{\infty} \frac{1}{h} \int_{x}^{x+h}\left(|T f|(y)-\frac{1}{h} \int_{x+h}^{x+2 h}|T f|\right)^{+} d y \leq C(w)\|f w\|_{\infty}
$$

for every $h>0, x \in \mathbb{R}$. Let $1<p<\infty$ and $w \in A_{p}^{+}$. Then

$$
\int|T f|^{p} w \leq C \int|f|^{p} w
$$

provided the left hand side is finite.

Here is the estimate we get for the operator $S$.

Theorem 2.4. Assume that $w$ is such that $w^{-1} \in A_{1}^{-}$. Then there exists a constant $C(w)$, which may depend on $w$, such that for every $h>0$ and $x_{0} \in \mathbb{R}$,

$$
\left\|w \chi_{\left[x_{0}-h, x_{0}\right]}\right\|_{\infty} \frac{1}{h} \int_{x_{0}}^{x_{0}+h}\left|S f(x)-S\left(f \chi_{\left(x_{0}+8 h, \infty\right)}\right)\left(x_{0}\right)\right| d x \leq C(w)\|f w\|_{\infty} .
$$

Proof. Given a function $f$ we define $f_{1}=f \chi_{\left(x_{0}, x_{0}+8 h\right)}, f_{2}=f \chi_{\left(x_{0}+8 h, \infty\right)}$. Then by using the linear operator introduced in Definition 1.1, we have

$$
\begin{aligned}
\frac{1}{h} \int_{x_{0}}^{x_{0}+h}\left|S f(x)-S f_{2}\left(x_{0}\right)\right| d x= & \frac{1}{h} \int_{x_{0}}^{x_{0}+h}\left|\|U f(x)\|_{\ell^{2}}-\left\|U f_{2}\left(x_{0}\right)\right\|_{\ell^{2}}\right| d x \\
\leq & \frac{1}{h} \int_{x_{0}}^{x_{0}+h}\left\|U f(x)-U f_{2}\left(x_{0}\right)\right\|_{\ell^{2}} d x \\
\leq & \frac{1}{h} \int_{x_{0}}^{x_{0}+h}\left\|U f_{1}(x)\right\|_{\ell^{2}} d x \\
& +\frac{1}{h} \int_{x_{0}}^{x_{0}+h}\left\|U f_{2}(x)-U f_{2}\left(x_{0}\right)\right\|_{\ell^{2}} d x \\
= & B_{1}+B_{2} .
\end{aligned}
$$

We choose $t>1$ sufficiently close to 1 such that:

(i) $\left(w^{-1}\right)^{t} \in A_{1}^{-}$,

(ii) there exists $s$ in the range $2<s<\infty$ such that $1 / 2+1 / s+1 / t=1$. 
Observe that in this case $1 / s+1 / t=1 / 2$. Then as the operator $S$ is bounded in $L^{p}(\mathbb{R})$ for every $p$ in the range $1<p<\infty$, we have

$$
\begin{aligned}
B_{1} & \leq\left(\frac{1}{h} \int_{x_{0}}^{x_{0}+h}\left\|U\left(f_{1}\right)(x)\right\|_{\ell^{2}}^{t} d x\right)^{1 / t}=\left(\frac{1}{h} \int_{x_{0}}^{x_{0}+h}\left|S f_{1}\right|^{t}\right)^{1 / t} \\
& \leq C\left(\frac{1}{h} \int_{x_{0}}^{x_{0}+8 h}|f|^{t} d x\right)^{1 / t} \\
& \leq C\|f w\|_{\infty}\left(\frac{1}{h} \int_{x_{0}}^{x_{0}+8 h}\left(w^{-1}(x)\right)^{t} d x\right)^{1 / t} .
\end{aligned}
$$

Since $w^{-t} \in A_{1}^{-}$we have

$$
\left\|w \chi_{\left(x_{0}-h, x_{0}\right)}\right\|_{\infty} B_{1} \leq\|f w\|_{\infty}\left\|w \chi_{\left(x_{0}-h, x_{0}\right)}\right\|_{\infty}\left(\frac{1}{h} \int_{x_{0}}^{x_{0}+8 h}\left(w^{-1}\right)^{t}\right)^{1 / t} \leq C .
$$

In order to bound $B_{2}$, we consider an integer $i$ such that $2^{i} \leq h<2^{i+1}$. If we use Hölder's inequality and our smoothness condition, we get

$$
\begin{aligned}
\left\|U f_{2}(x)-U f_{2}\left(x_{0}\right)\right\|_{\ell^{2}} & \leq \int_{x_{0}+8 h}^{\infty}\left\|K(x-y)-K\left(x_{0}-y\right)\right\|_{\ell^{2}}|f(y)| d y \\
\leq & \sum_{j=i+3}^{\infty}\left(\int_{x_{0}+2^{j}}^{x_{0}+2^{j+1}}\left\|K(x-y)-K\left(x_{0}-y\right)\right\|_{\ell^{2}}^{2} d y\right)^{1 / 2} \\
& \times\left(\int_{x_{0}+2^{j}}^{x_{0}+2^{j+1}} w^{-t}\right)^{1 / t}\left(\int_{x_{0}+2^{j}}^{x_{0}+2^{j+1}} f^{s} w^{s}\right)^{1 / s} \\
\leq & C\|f w\|_{\infty} \sum_{j>i+3} 2^{i / 2} 2^{-j} 2^{j / s}\left(\int_{x_{0}}^{x_{0}+2^{j+1}} w^{-t}\right)^{1 / t} .
\end{aligned}
$$

Therefore as $t$ was chosen in such a way that $w^{-t} \in A_{1}^{-}$and $1 / s+1 / t=1 / 2$, we have

$$
\begin{aligned}
\left\|w \chi_{\left(x_{0}-h, x_{0}\right)}\right\|_{\infty} B_{2} & \leq C\|f w\|_{\infty} \sum_{j>i+3} 2^{i / 2} 2^{-j} 2^{j / s} 2^{j / t} \\
& \leq C\|f w\|_{\infty} \sum_{j>i+3} 2^{i / 2} 2^{-j / 2} \leq C\|f w\|_{\infty} .
\end{aligned}
$$

In order to check that the hypotheses of the extrapolation theorem are 
satisfied, we just look at the following inequalities:

$$
\begin{aligned}
\frac{1}{h} \int_{x}^{x+h}\left(S f(y)-\frac{1}{h} \int_{x+h}^{x+2 h} S f\right)^{+} d y \leq & \frac{1}{h} \int_{x}^{x+h}\left|S f(y)-S\left(f \chi_{(x+8 h, \infty)}\right)(x)\right| d y \\
& \left.+\frac{1}{h} \int_{x+h}^{x+2 h} \mid S f(y)-S\left(f \chi_{(x+8 h, \infty)}\right)(x)\right) \mid d y \\
\leq & \left.C \frac{1}{h} \int_{x}^{x+2 h} \mid S f(y)-S\left(f \chi_{(x+8 h, \infty)}\right)(x)\right) \mid d y
\end{aligned}
$$

Therefore $S$ maps $L^{p}(w)$ into itself if $w \in A_{p}^{+}$, provided $p>1$.

For the case $p=1$ we obtain weak type. The proof will use the following lemma:

LEMMA 2.5. Let a be a function supported on $I=\left(x^{*}, x^{*}+h\right)$ such that $\int_{I} a(y) d y=0$. For any $w \in A_{1}^{+}$there exists $C$ depending only on $w$ so that

$$
\int_{y<x^{*}-2 h} S a(y) w(y) d y \leq C \int_{I}|a(y)| w(y) d y .
$$

Proof. It is enough to prove the following

Claim. Let a be a function supported on $I=\left(x^{*}, x^{*}+2^{i}\right)$ such that $\int_{I} a(y) d y=0$. For any $w \in A_{1}^{+}$there exists $C$ depending only on $w$ so that

$$
\int_{y<x^{*}-2^{i}} S a(y) w(y) d y \leq C \int_{I}|a(y)| w(y) d y .
$$

Observe that if the claim is true, given $h$ we choose $i$ such that $2^{i-1} \leq$ $h<2^{i}$; then $\int_{y<x^{*}-2^{i}} a(y) d y=0$ and $\left\{y<x^{*}-2 h\right\} \subset\left\{y<x^{*}-2^{i}\right\}$.

Now we shall prove the claim. For $k=0,1,2 \ldots$ let $x_{k}=x^{*}-2^{k+i}$. Then

$$
\int_{y<x^{*}-2^{i}} S a(y) w(y) d y=\sum_{k=1}^{\infty} \int_{x_{k}}^{x_{k-1}} S a(y) w(y) d y .
$$

Now if $x \in I_{k}=\left[x_{k}, x_{k-1}\right]$ and $j>k+i$ then

$$
A_{j} a(x)=\frac{1}{2^{j}} \int_{x}^{x+2^{j}} a(y) d y=\frac{1}{2^{j}} \int_{x^{*}}^{x+2^{j}} a(y) d y=\frac{1}{2^{j}} \int_{I} a(y) d y=0,
$$

because $x+2^{j} \geq x^{*}-2^{k+i}+2^{k+i+1} \geq x^{*}+2^{i}$. But if $j<k+i$ then $x+2^{j} \leq x^{*}-2^{k+i-1}+2^{k+i-1}<x^{*}$ and again $A_{j} a(x)=0$. If $j=k+i$ and $x_{k}+2^{i}<x$ then $x+2^{j} \geq x^{*}-2^{k+i}+2^{i}+2^{k+i}=x^{*}+2^{i}$, and $A_{j}(x)=0$. In other words, on each $I_{k}, S f$ is zero except on the subinterval $\left(x_{k}, x_{k}+2^{i}\right)$, 
and on that interval it is less than or equal to $\left(C / 2^{k+i}\right) \int|a(y)| d y$. Now

$$
\int_{y<x^{*}-2^{i}} S a(y) w(y) d y \leq C \sum_{k=1}^{\infty} \frac{1}{2^{k+i}} \int_{x_{k}}^{x_{k}+2^{i}} w(y) \int_{I}|a(z)| d z d y .
$$

If, for each $k$, we use the fact that $w^{r} \in A_{1}^{+}$for some $r>1$, we may write

$$
\begin{aligned}
\int_{x_{k}}^{x_{k}+2^{i}} w(y) \int_{I}|a(z)| d z d y & \leq\left(\int_{x_{k}}^{x_{k}+2^{i}} w(y)^{r} d y\right)^{1 / r} 2^{i / r^{\prime}} \int_{I}|a(z)| d z \\
& \leq\left(\int_{x_{k}}^{x^{*}} w(y)^{r} d y\right)^{1 / r} 2^{i / r^{\prime}} \int|a(z)| d z \\
& \leq C 2^{i / r^{\prime}} 2^{(k+i) / r} \int_{I}|a(z)| w(z) d z
\end{aligned}
$$

where the constant $C$ depends only on $w$. If we sum over $k$ we have

$$
\begin{aligned}
\int_{y<x^{*}-2^{i}} S a(y) w(y) d y & \leq \sum_{k=1}^{\infty} C \frac{1}{2^{k+i}} 2^{(k+i) / r} 2^{i / r^{\prime}} \int_{I}|a(y)| w(y) d y \\
& \leq C \int_{I}|a(y)| w(y) d y .
\end{aligned}
$$

Theorem 2.6. Let $w \in A_{1}^{+}$. Then there exists $C$, depending only on $w$, so that for any $\lambda>0$,

$$
\int_{\{x: S f(x)>\lambda\}} w(x) d x \leq \frac{C}{\lambda} \int|f(x)| w(x) d x .
$$

Proof. Let $O_{\lambda}=\left\{x: M^{+} f(x)>\lambda\right\}$. It is well known [8] that if $I_{i}$ are the connected components of $O_{\lambda}$, then $\lambda=\left(1 /\left|I_{i}\right|\right) \int_{I_{i}} f=f_{I_{i}}$. We decompose $f$ as

$$
f=f \chi_{\mathbb{R} \backslash O_{\lambda}}+\sum f_{I_{i}} \chi_{I_{i}}+\sum\left(f-f_{I_{i}}\right) \chi_{I_{i}}
$$

As usual $f \chi_{\mathbb{R} \backslash O_{\lambda}}+\sum f_{I_{i}} \chi_{I_{i}}$ will be denoted by $g$, and $\sum\left(f-f_{I_{i}}\right) \chi_{I_{i}}=\sum b_{i}$ by $b$. Observe that each $b_{i}$ has support on $I_{i}$ and average zero. Now,

$$
\begin{aligned}
& \int_{\mathbb{R}}|g(y)| w(y) d y \leq \int_{\mathbb{R} \backslash O_{\lambda}}|f(y)| w(y) d y+\sum w\left(I_{i}\right) f_{I_{i}} \\
& \quad=\int_{\mathbb{R} \backslash O_{\lambda}}|f(y)| w(y) d y+\lambda \sum w\left(I_{i}\right)=\int_{\mathbb{R} \backslash O_{\lambda}}|f(y)| w(y) d y+\lambda w\left(O_{\lambda}\right) \\
& \quad \leq C \int|f| w,
\end{aligned}
$$

because the operator $M^{+} f$ is of weak type $(1,1)$ with respect to $w$. 
For each interval $I=(b, c)$, denote by $2 I$ the interval $(b-2 c, c)$. We also denote by $\widetilde{O}_{\lambda}$ the union of all the intervals $2 I_{i}, I_{i}$ being the connected components of $O_{\lambda}$. The one-sided doubling property of the weight (see the comments at the beginning of Section 2) gives

$$
w\left(\widetilde{O}_{\lambda}\right)=w\left(\bigcup_{i} 2 I_{i}\right) \leq C w\left(O_{\lambda}\right) \leq \frac{C}{\lambda} \int|f(y)| w(y) d y .
$$

Observe that

$$
\begin{aligned}
w\{x: S f(x)>\lambda\} \leq & w\{x: S g(x)>\lambda / 2\}+w\left(\widetilde{O}_{\lambda}\right) \\
& +w\left\{x \notin \widetilde{O}_{\lambda}: S b(x)>\lambda / 2\right\} .
\end{aligned}
$$

The second term is already known to be bounded by $(C / \lambda) \int|f(x)| w(x) d x$. Since $S$ is a bounded operator in $L^{2}(w)$, and condition $A_{1}^{+}$implies condition $A_{p}^{+}$for any $p>1$, we have

$$
\begin{aligned}
w\{x: S g(x)>\lambda / 2\} & \leq \frac{C}{\lambda^{2}} \int(S g(y))^{2} w(y) d y \leq \frac{C}{\lambda^{2}} \int|g(y)|^{2} w(y) d y \\
& \leq \frac{C}{\lambda} \int|g(y)| w(y) d y \leq \frac{C}{\lambda} \int|f(y)| w(y) d y .
\end{aligned}
$$

In the last two inequalities we have used $|g| \leq \lambda$ and $\int|g| w \leq C \int|f| w$. Finally for the third term by using the preceding lemma and the one-sided nature of the operator $S$, we have

$$
\begin{aligned}
w\left\{x \notin \widetilde{O}_{\lambda}: S b(x)>\lambda / 2\right\} & \leq \frac{C}{\lambda} \int_{\mathbb{R} \backslash \widetilde{O}_{\lambda}} S b(x) w(x) d x \\
& \leq \frac{C}{\lambda} \sum_{j} \int_{\mathbb{R} \backslash 2 I_{i}} S b_{i}(x) w(x) d x \\
& \leq \sum_{i} \int_{I_{i}}\left|b_{i}(x)\right| w(x) d x .
\end{aligned}
$$

But since the $I_{i}$ 's are disjoint and $b(x)=b_{i}(x)$ on each $I_{i}$, the last term is bounded by

$$
\int|b(x)| w(x) d x=\int|f(x)-g(x)| w(x) d x \leq C \int|f(x)| w(x) d x .
$$

Now we shall prove the converses of the last theorems.

Theorem 2.7. Assume that for some $p \geq 1$ there exists a constant $C_{p}$ so that

$$
\int_{\{x: S f(x)>\lambda\}} w \leq \frac{C_{p}}{\lambda^{p}} \int|f|^{p} w .
$$

Then $w$ satisfies condition $A_{p}^{+}$, and the constant in the condition depends only on the constant $C_{p}$. 
Proof. We shall prove that the weight $w$ satisfies condition $\widetilde{A}_{p}^{+}$(see Remark 2.3). Let $p>1$. Let $i$ be an integer such that $2^{i}<h<2^{i+1}$. Let $f=w^{1-p^{\prime}} \chi_{(b, b+h)}$. If $x \in(b-2 h, b-h)$ then $A_{i} f(x)=0$ and $A_{i+3} f(x)=$ $\left(1 / 2^{i+3}\right) \int_{b}^{b+h} w^{1-p^{\prime}}$. If we choose $\lambda=\left(1 / 2^{i+3}\right) \int_{b}^{b+h} w^{1-p^{\prime}}$, we have

$$
\begin{aligned}
\lambda=A_{i+3} f(x) \leq & \left|A_{i+3} f(x)-A_{i+2} f(x)\right|+\left|A_{i+2} f(x)-A_{i+1} f(x)\right| \\
& +\left|A_{i+1} f(x)-A_{i} f(x)\right| \\
\leq & C S f(x) .
\end{aligned}
$$

This means that $(b-2 h, b-h) \subset\{x: S f(x)>\lambda\}$, and then

$$
\int_{b-2 h}^{b-h} w \leq C h^{p}\left(\int_{b}^{b+h} w^{1-p^{\prime}}\right)^{1-p}
$$

which is $\widetilde{A}_{p}^{+}$. We have thus proved that for $p>1$ the operator $S$ is bounded on $L^{p}(w)$ if and only if $w$ satisfies $A_{p}^{+}$. If $p=1$ and $b$ is a Lebesgue point for $w$, we consider the interval $\left(b, b+2^{i}\right)$ and $f=\chi_{(b, b+h)}$, where $h<2^{i}$. It is clear that if $x \in\left(b-2^{i+1}, b-2^{i}\right)$ then $A_{i} f(x)=0$, while $A_{i+2} f(x)=$ $\left(1 / 2^{i+2}\right) h$. It follows that $S f(x)>C h / 2^{i}$, and so $\left(b-2^{i+1}, b-2^{i}\right) \subset\{x$ : $\left.S f(x)>h / 2^{i}\right\}$. Therefore

$$
w\left(b-2^{i+1}, b-2^{i}\right) \leq C \frac{2^{i}}{h} \int_{b}^{b+h} w
$$

It follows that

$$
\frac{1}{2^{i}} w\left(b-2^{i+1}, b-2^{i}\right) \leq C w(b) .
$$

If now $I=(a, b)$ is any interval of length $2^{j}$ we define $x_{0}=a$ and for $k \geq 1$, $x_{k}=\left(x_{k-1}+b\right) / 2$. We may then write

$$
\int_{I} w=\sum \int_{x_{k}}^{x_{k+1}} w \leq C w(b) \sum\left(x_{k+1}-x_{k}\right)=C w(b)(b-a),
$$

which is $A_{1}^{+}$.

REMARK 2.8. It is easy to see that the same methods prove that the operator $S_{1}$ defined in the introduction maps $L^{p}(w)$ into itself if and only if the weight $w$ satisfies condition $A_{p}^{+}$, but restricted to intervals of length less than one.

\section{The action of the one-sided maximal operator on BMO func-} tions. It is easy to prove that for certain functions $f$ in BMO the maximal operator $M^{+} f$ is infinite at every point. Take for example $f(x)=\log ^{+} x$. In fact results similar to Propositions 1.8 and 1.9 can be proved in this case; we leave the details to the reader. 
On the other hand it is extremely easy to prove that if a function $f$ is in BMO and $M^{+} f(x)<\infty$ for a.e. $x$ then $M^{+} f \in$ BMO. This fact is parallel to the corresponding result for the Hardy-Littlewood maximal operator in [2], but it does not follow from the fact that $M^{+} f(x) \leq M f(x)$, because $g \in \mathrm{BMO}$ and $0 \leq f \leq g$ do not imply $f \in$ BMO. We need the following lemma, whose detailed and easy proof is left to the reader.

Lemma 3.9. Let $I=\left(x_{0}, x_{0}+h\right), k>h$. Then

$$
\int_{x_{0}+k}^{x_{0}+k+h}\left|f(x)-f_{I}\right| d x \leq C k\|f\|_{\mathrm{BMO}} .
$$

Theorem 3.10. If $f \in \mathrm{BMO}$ then either $M^{+} f(x)=\infty$ for a.e. $x$ or $M^{+} f(x)<\infty$ for a.e. $x$. In the second case $M^{+} f \in \mathrm{BMO}$ and $\left\|M^{+} f\right\|_{\mathrm{BMO}}$ $\leq C\|f\|_{\text {BMO }}$.

Proof. Fix $x_{0}$ and $h>0$. Let $I=\left(x_{0}, x_{0}+h\right)$. We decompose $f$ as $f=$ $f_{1}+f_{2}$, where $f_{1}(x)=\left(f(x)-f_{I}\right) \chi_{\left(x_{0}, x_{0}+2 h\right)}(x)$ and $f_{2}(x)=f(x)-f_{1}(x)$. Since $M^{+} f(x)$ and $M^{+} f_{2}\left(x_{0}\right)$ are finite, we may write

$$
\begin{aligned}
& \frac{1}{h} \int_{x_{0}}^{x_{0}+h}\left|M^{+} f(x)-M^{+} f_{2}\left(x_{0}\right)\right| d x \\
& \quad \leq \frac{1}{h} \int_{I} M^{+} f_{1}(x) d x+\frac{1}{h} \int_{I} \sup _{k>0}\left|\frac{1}{k} \int_{x}^{x+k} f_{2}(y) d y-\frac{1}{k} \int_{x_{0}}^{x_{0}+k} f_{2}(y) d y\right| d x \\
& \quad=B_{1}+B_{2} .
\end{aligned}
$$

If we use Hölder's inequality, the fact that the operator $M^{+}$is bounded in $L^{p}$ for any $p>1$, and the John-Nirenberg theorem, we get $B_{1} \leq C\|f\|_{\mathrm{BMO}}$.

Now we shall analyze $B_{2}$. Due to the one-sided nature of the operator $M^{+}$, we can substitute $f_{2}$ by $g_{2}(x)=\left(f(x)-f_{I}\right) \chi_{\left(x_{0}+2 h, \infty\right)}(x)$. Now for each $k>0$ it is clear that

$$
\frac{1}{k} \int_{x}^{x+k} g_{2}(y) d y-\int_{x_{0}}^{x_{0}+k} g_{2}(y) d y
$$

is 0 unless $k>h$ and in this case

$$
\frac{1}{k}\left|\int_{x}^{x+k} g_{2}-\int_{x_{0}}^{x_{0}+k} g_{2}\right| \leq \frac{1}{k} \int_{x_{0}+k}^{x_{0}+h+k}\left|g_{2}(y)\right| d y .
$$

But since $g_{2}(y)=\left(f(y)-f_{I}\right) \chi_{\left(x_{0}+2 h, \infty\right)}(y)$, the last lemma tells us that

$$
B_{2} \leq C\|f\|_{\mathrm{BMO}}
$$




\section{References}

[1] H. Aimar, L. Forzani and F. J. Martín-Reyes, On weighted inequalities for one-sided singular integrals, Proc. Amer. Math. Soc. 125 (1997), 2057-2064.

[2] C. Bennett, R. A. DeVore and R. Sharpley, Weak- $L^{\infty}$ and BMO, Ann. of Math. 113 (1981), 601-611.

[3] R. Jones, R. Kaufman, J. M. Rosenblatt and M. Wierld, Oscillation in ergodic theory, Ergodic Theory Dynam. Systems 18 (1998), 889-935.

[4] R. L. Jones, I. V. Ostrovskii and J. M. Rosenblatt, Square functions in ergodic theory, ibid. 16 (1996), 267-305.

[5] R. A. Macías and M. S. Riveros, One-sided extrapolation at infinity and singular integrals, Proc. Roy. Soc. Edinburgh Sect. A 130 (2000), 1081-1102.

[6] M. S. Riveros and A. de la Torre, On the best ranges for $A_{p}^{+}$and $\mathrm{RH}_{r}^{+}$, Czechoslovak Math. J. 51 (126) (20001), 285-301.

[7] J. L. Rubio de Francia, F. J. Ruiz and J. L. Torrea, Calderón-Zygmund theory for vector-valued functions, Adv. Math. 62 (1986), 7-48.

[8] E. Sawyer, Weighted inequalities for the one-sided Hardy-Littlewood maximal functions, Trans. Amer. Math. Soc. 297 (1986), 53-61.

Departamento de Análisis Matemático

Departamento de Matemáticas

Facultad de Ciencias

Universidad de Málaga

Universidad Autónoma de Madrid

29071 Málaga, Spain

28049 Madrid, Spain

E-mail: torre@anamat.cie.uma.es

E-mail: joseluis.torrea@uam.es

Received March 1, 2002

Revised version November 14, 2002 